\title{
Severe Hypertriglyceridaemia Leading to Factitious Hypobicarbonataemia
}

\author{
Sasmit Roy ${ }^{1}$, Mohammed Ashraf ${ }^{1}$, Satbyul Sophia Kang ${ }^{1}$, Raul Ayala², Sreedhar Adapa ${ }^{3}$ \\ ${ }^{1}$ Department of Internal Medicine, Centra Health, Lynchburg, VA, USA \\ ${ }^{2}$ Department of Internal Medicine, Adventist Health, Hanford, CA, USA \\ ${ }^{3}$ Department of Internal Medicine, Kaweah Health Medical Center, Visalia, CA, USA
}

Received: 03/11/2021

Accepted: $10 / 12 / 2021$

Published: 24/12/2021

\begin{abstract}
How to cite this article: Roy S, Ashraf M, Kang SS, Ayala R, Adapa S. Severe hypertriglyceridaemia leading to factitious hypobicarbonataemia. EJCRIM
\end{abstract} 2021;8: doi:10.12890/2021_003046.

Conflicts of Interests: The authors declare there are no competing interests.

This article is licensed under a Commons Attribution Non-Commercial 4.0 License

\section{ABSTRACT}

Anion gap metabolic acidosis is a laboratory finding commonly encountered in patients with sepsis, diabetic ketoacidosis, acute kidney injury and toxic alcohol ingestion. Serum blood chemistry assessment detects this abnormality. However, this can be falsely low in situations of high triglyceride levels due to lipid interference with measurement of the bicarbonate levels and through volume displacement by these large molecules. Arterial blood gas analysis and a lipid panel are required to confirm accurate bicarbonate levels. Clinicians handling acidbase disorders in hospitalized patients need to be aware of this spurious laboratory value to avoid unnecessary tests and to determine accurate total bicarbonate levels.

\section{LEARNING POINTS}

- $\quad$ Elevated triglycerides can cause spurious low blood bicarbonate levels.

- High anion gap metabolic acidosis is associated with serious clinical illness which can lead to a battery of diagnoses and therapeutics.

- Accurate knowledge of this condition with falsely low bicarbonate levels will prevent unnecessary medical work-up and guide prompt treatment.

\section{KEYWORDS}

Factitious hypobicarbonataemia, metabolic acidosis

\section{INTRODUCTION}

Metabolic acidosis is a very common finding in clinical settings, especially in instances of sepsis, alcohol overdose, diabetic ketoacidosis, acute kidney injury and diarrhoea. Anion gap metabolic acidosis is associated with debilitating conditions like septic shock, severe lactic acidosis, alcohol overdose and diabetic ketoacidosis which can be life-threatening if not identified early and treated accordingly. However, it can be falsely reported in basic metabolic chemistry assessment without apparent clinical irregularities in conditions associated with paraproteinaemia or hyperlipidaemia. Knowledge of these spurious conditions needs to be reinforced in physicians to avoid unnecessary extensive tests and empiric treatments. We present a similar case of falsely low serum bicarbonate levels (CO2) with normal arterial blood gas bicarbonate levels in a patient with elevated triglyceride (TG) levels which may have interfered with the accurate measurement of serum bicarbonate. 


\section{CASE DESCRIPTION}

A 46-year-old Caucasian male with a history of chronic myeloid leukaemia in remission, hypertension and hyperlipidaemia presented to a regional Emergency Department (ED) with a severe headache that had been ongoing for the previous $2-3$ weeks. The headache was diffuse in nature and associated with blurry vision. He had no associated diarrhoea or vomiting before the presentation. He had no symptoms of chest pain, shortness of breath, nausea, vomiting, chills, rigor, abdominal pain or any urinary complaints. He had no family history of lipidrelated disorders. His home medications included amlodipine 5 milligrams (mg) daily, lisinopril 10 mg daily and atorvastatin 80 mg daily. He was found to have elevated blood pressure (BP) of 185/121 mmHg, a heart rate of 112 beats/min, a respiratory rate of 18/min and oxygen saturation of $98 \%$ on room air. Physical examination was unremarkable. He was started on treatment with intravenous (IV) labetalol and hydralazine. Laboratory work-up in the ED was remarkable for a serum CO2 level of $12 \mathrm{mmol} / \mathrm{I}$ with an elevated anion gap of $20 \mathrm{mmol} / \mathrm{l}$. His other electrolytes and renal function were unremarkable. His complete blood count was unremarkable. Computed tomography (CT) of the head without contrast revealed no acute intracranial pathology. He was given 1 litre of isotonic sodium bicarbonate (150 mEq of sodium bicarbonate in 1 litre of 5\% dextrose water) initially to correct his low serum bicarbonate levels. When the infusion did not correct low serum bicarbonate on repeat labs, an arterial blood gas was obtained, which showed arterial pH of 7.43, bicarbonate levels of 25 and pCO2 levels of 38. Simultaneous serum chemistry demonstrated low CO2 at $12 \mathrm{mmol} / \mathrm{I}$. He continued to have intractable headaches despite IV narcotics given in the ED.

Due to the history of hyperlipidaemia, a lipid panel was conducted which returned an elevated total cholesterol (TC) of $11.5 \mathrm{mmol} / \mathrm{I}$, markedly elevated TGs of $26.7 \mathrm{mmol} / \mathrm{l}$ and a low-density lipoprotein (LDL) level of $0.67 \mathrm{mmol} / \mathrm{I}$ with elevated very-low-density lipoprotein (VLDL) of $9.8 \mathrm{mmol} / \mathrm{l}$ and low high-density lipoprotein (HDL) levels of $0.5 \mathrm{mmol} / \mathrm{I}$. The lipid panel carried out 6 months prior showed TC of $5.4 \mathrm{mmol} / \mathrm{I}$, TG of $6.6 \mathrm{mmol} / \mathrm{I}$ and VLDL of $2.4 \mathrm{mmol} / \mathrm{I}$. The patient was started on fenofibrate $200 \mathrm{mg}$ daily along with a 4 gram daily dose of omega-3 fatty acids.

Serum amylase and lipase levels were measured to rule out pancreatitis which returned normal. Thyroid stimulating hormone levels were unremarkable.

Over the next 3 days of his initial admission, the patient's work-up included negative magnetic resonance imaging/magnetic resonance angiography of the head and neck. The headaches resolved with acetaminophen/butalbital combination along with control of elevated BP. BP was eventually controlled on adjusting the medications to oral antihypertensive agents, metoprolol 50 mg daily, amlodipine 10 mg daily and lisinopril $40 \mathrm{mg}$ daily.

\section{DISCUSSION}

Metabolic acidosis is a pathological process stemming from dysregulation of the finely regulated acid-base equilibrium of the body when there is either reduced bicarbonate excretion or an increased concentration of hydrogen ions in the body. Using the classic HendersonHasselbalch equation (Fig. 1), bicarbonate in the arterial blood gas can be calculated, whereas serum bicarbonate is measured by calculating the concentration of total $\mathrm{CO} 2$ in the blood which includes bicarbonate ( $\mathrm{HCO} 3-)$, carbonic acid ( $\mathrm{H} 2 \mathrm{CO} 3$ ) and dissolved $\mathrm{CO} 2$ in blood.

\section{The Henderson-Hasselbalch Equation $(\mathrm{H}-\mathrm{H})$}

$\mathrm{H}-\mathrm{H}$ equation mathematically illustrates how the $\mathrm{pH}$ of a solution is influenced by the $\mathrm{HCO}_{3}{ }^{-}$to $\mathrm{H}_{2} \mathrm{CO}_{3}$ ratio (the bicarbonate buffer system); the base to acid ratio

$\mathrm{H}-\mathrm{H}$ equation is written as follows:

$$
\mathrm{pH}=\mathrm{pK}+\log \frac{\left[\mathrm{HCO}_{3}^{-}\right] \text {(base) }}{\left[\mathrm{H}_{2} \mathrm{CO}_{3}\right] \text { (acid) }}
$$

$\mathrm{pK}$ is derived from the dissociation constant of the acid portion of the buffer combination

pK is 6:1 and, under normal conditions, the $\mathrm{HCO}_{3}{ }^{-}$to $\mathrm{H}_{2} \mathrm{CO}_{3}$ ratio is 20:1

Clinically, the dissolved $\mathrm{CO}_{2}\left(\mathrm{PCO}_{2} \times 0.03\right)$ can be used for the denominator of the $\mathrm{H}-\mathrm{H}$ equations, instead of the $\mathrm{H}_{2} \mathrm{CO}_{3}$

This is possible since the dissolved carbon dioxide is in equilibrium with, and directly proportional to, the blood $\left[\mathrm{H}_{2} \mathrm{CO}_{3}\right]$, the $\mathrm{PaCO} 2$ is easily measured via blood gas analysis and can easily be converted to $\mathrm{mmol} / \mathrm{L}$ (same as $\mathrm{mEq} / \mathrm{L}$ ).

Thus, the $\mathrm{H}-\mathrm{H}$ equation can be written as follows:

$$
\mathrm{pH}=\mathrm{pK}+\log \frac{\left[\mathrm{HCO}_{3}^{-}\right]}{\left[\mathrm{PCO}_{2} \times 0.03\right]}
$$


This discrepancy is mainly due to lipid interference in some analyzers (for example, Abbott Architect) ${ }^{[1]}$.

This condition of falsely low bicarbonate levels, clinically known as pseudohypobicarbonataemia (PHB), has been reported in a few instances ${ }^{[2]}$.

Analyzers usually measure serum CO2 levels by the phosphoenolpyruvate (PEP) carboxylase reaction where PEP and CO2 are converted to oxaloacetate and phosphate, and then reduced to malate with simultaneous nicotinamide adenine dinucleotide oxidation. This decreased absorbance is proportional to the $\mathrm{CO} 2$ content in blood which can be disrupted by TGs through absorbing light ${ }^{[3]}$.

Lipids also disrupt the accurate measurement through space-occupying lesions by volume displacement, lowering the aqueous phase of the sample and thereby falsely reducing the levels of potassium, chloride, sodium and so on, and thus the spurious low values are observed ${ }^{[4]}$. Increased turbidity of the sample is incurred through a high level of circulating TG levels and thus the false laboratory findings ${ }^{[5]}$.

It has been reported that an indirect ion-specific electrode method to estimate the total carbon dioxide levels in blood can avoid this error ${ }^{[2]}$. Our patient had no clinical evidence of conditions that could have led to metabolic acidosis. Timely diagnosis of hypertriglyceridaemia and prompt initiation of lipid-lowering agents helped avoid unnecessary tests and therapies. The discrepancy between arterial blood gas and venous serum chemistry $\mathrm{CO} 2$ should raise suspicion of hypertriglyceridaemia. Clinicians need to be aware of this association and check lipid panels in instances of confusing serum bicarbonate levels.

\section{REFERENCES}

Goldwasser P, Manjappa NG, Luhrs CA, Barth RH. Pseudohypobicarbonatemia caused by an endogenous assay interferent: a new entity. Am J Kidney Dis 2011;58(4):617-620. Stein H. Spuriously low serum bicarbonate levels in patients with hyperlipidemia: a report of 4 cases. Am J Kidney Dis 2019;73(1):131-133.

Varghese V, Griener D, Wu Q, Velez JC. Pseudohypobicarbonatemia in severe hypertriglyceridemia. Am J Kidney Dis 2020;76(4):601-603.

Rifkin SI, Shaub B. Factitious hypobicarbonatemia associated with profound hyperlipidemia. Ren Fail 2014;36(7):1155-1157.

Dana AP, Kazory A. Spurious low serum bicarbonate level due to severe hypertriglyceridemia: a clinical challenge. Am J Med 2020;133(6):e306-e307. 